\title{
Reflectance anisotropy spectroscopy of strain-engineered
}

\section{GaAsBi alloys}

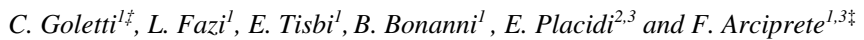 \\ ${ }^{1}$ Dipartimento di Fisica, Università di Roma "Tor Vergata", Via della Ricerca Scientifica 1 \\ I-00133 Roma, Italy \\ ${ }^{2}$ Dipartimento di Fisica, Università di Roma “La Sapienza”, Piazzale Aldo Moro 2 \\ I-00185 Roma, Italy \\ ${ }^{3}$ Istituto di Struttura della Materia, Consiglio Nazionale delle Ricerche, Via Fosso del Cavaliere 100 \\ I-00133, Roma, Italy
}

\begin{abstract}
In this paper we present results obtained by an optical technique, namely Reflectance Anisotropy Spectroscopy (RAS), applied to a series of $\mathrm{GaAs}_{1-\mathrm{x}} \mathrm{Bi}_{\mathrm{x}}$ samples grown by Molecular Beam Epitaxy (MBE) under different strain conditions with increasing concentration of $\mathrm{Bi}$, up to the higher value of about $7 \%$. The epitaxial buffer layers for the growing $\mathrm{GaAs}_{1-\mathrm{x}} \mathrm{Bi}_{\mathrm{x}}$ layer were prepared with either a compressive strain (as it is commonly done) or a tensile strain: the latter case has been proven to be a strategy that allows to obtain a better crystalline quality [E. Tisbi, E. Placidi, R. Magri, P. Prosposito, R. Francini, A. Zaganelli, S. Cecchi, E. Zallo, R. Calarco, E. Luna, J. Honolka, M. Vondráček, S. Colonna and F. Arciprete, Phys. Rev. Applied 14, 014028 (2020)]. A characteristic, well defined anisotropy signal below $2.5 \mathrm{eV}$ is demonstrated to be connected to the presence of $\mathrm{Bi}$ and in particular to the strain produced in the sub-surface region by the voluminous $\mathrm{Bi}$ atoms. The amplitude of this signal directly relates to the Bi quantity, while its sign gives information about the local clustering/ordering of $\mathrm{Bi}$ atoms in the grown sample. We conclude that the detailed interpretation of RAS signatures and the knowledge of their origin offer the opportunity to utilize this technique to follow in real time the GaAsBi growth either in MBE as well as in MOVPE (Metal Organic Vapour Phase Epitaxy) processes.
\end{abstract}

Corresponding author:

claudio.goletti@roma2.infn.it, arciprete@roma2.infn.it 
The increasing interest for gallium arsenic bismide alloys $\left(\mathrm{GaAs}_{1-\mathrm{x}} \mathrm{Bi}_{\mathrm{x}}\right)$ calls for a high level of $\mathrm{Bi}$ incorporation in the samples, as the red-shift of the band-gap ( $88 \mathrm{meV} / \% \mathrm{Bi})$ and the related noteworthy properties depend directly upon that value [1-7]. Unfortunately, the quality of grown $\mathrm{GaAs}_{1-\mathrm{x}} \mathrm{Bi}_{\mathrm{x}}$ crystals rapidly decays with the amount of $\mathrm{Bi}$, imposing new and sometimes unconventional growth strategies to gain a higher incorporation of $\mathrm{Bi}$ in GaAs: low temperature of the substrate [8], variable flux of $\mathrm{Bi}$ and 1:1 As to Ga flux ratio for favoring the Bi incorporation [9]. The necessity to precisely control these parameters makes Molecular Beam Epitaxy (MBE) the preferred technique for the growth of GaAsBi, although MOVPE (Metal Organic Vapour Phase Epitaxy) is sometimes used. In the literature, values of Bi as high as $22 \%$ have been reported [10], being the result of these particular methods and recipes. A different strategy is based on a choice of the substrate such that the growing GaAsBi layer experiences a tensile strain (for a substrate unit cell larger than the GaAsBi one) instead of the more commonly used compressive strain (for a substrate unit cell smaller than the GaAsBi one, as for GaAsBi grown onto GaAs). It has been demonstrated that in this way a higher quality of the structural and optical properties at the same Bi incorporation is reached [11].

The usual procedure to evaluate the quality of the resulting sample is a post-growth and often $e x$-situ investigation of its structural, electronic and optical properties by X-Ray Diffraction (XRD), Raman, photoluminescence (PL), scanning probe microscopy. Reflection High Energy Electron Diffraction (RHEED) is commonly used in MBE to monitor the epitaxy of the growing layer: however, if an all-optical real-time monitoring of growth would be available, this could allow a complementary control and enable a real-time correction of the parameters that influence deposition, with the advantage of using low energy photons instead of high energy electrons. In MOVPE, this would cover the gap existing between growth and characterization of the samples, normally demanded to post-growth or even ex-situ techniques.

Reflectance Anisotropy Spectroscopy (RAS), a differential optical technique successfully applied to MBE and MOVPE growth of III-V compounds, could offer this opportunity [12]. In its pristine application, the exceptional sensitivity of RAS to optical anisotropy signals has been exploited to investigate the layerby-layer deposition during the growth, for example, of $\mathrm{GaAs}(001)$ [13], $\mathrm{GaP}(001)$ [14], $\operatorname{InGaAs}(001)$ [15], as well as to characterize clean surfaces of semiconductors [16-18], metals [19], insulating materials [20] and 
organic layers [21-23]. Very recently it has been applied to investigate the MOVPE growth of GaAsBi samples [24].

RAS has been generally applied to extract the layer or surface properties from the optical reflectance signal detected. In this case, the choice of a substrate having isotropic optical properties with respect to two orthogonal directions (usually coincident with symmetry directions in the surface plane) helps to disentangle the bulk contribution from the signal coming from the surface (having a different symmetry) [25]. An anisotropic bulk often represents a case to be avoided, for the detection of a large bulk signal and -in addition- for the lack of validity of the three-layer model commonly used to interpret the data and to extract the surface-related anisotropy [25]. In the experiments we present here, on the contrary, we have exploited the signal caused by the strain due to Bi inserted in the crystal lattice, while the surface signal was deliberately cancelled by contamination.

The RAS signal is defined as the ratio $\Delta R / R$ between the difference $\Delta R$ of the light intensity $R_{\alpha}$ and $\mathrm{R}_{\beta}$ reflected by the sample for a beam linearly polarized along the two orthogonal directions $\alpha$ and $\beta$ and their average $\mathrm{R}=\left(\mathrm{R}_{\alpha}+\mathrm{R}_{\beta}\right) / 2$, as a function of photon energy [12]:

$$
\Delta \mathrm{R} / \mathrm{R}=2 \frac{\mathrm{R}_{\alpha}-\mathrm{R}_{\beta}}{\mathrm{R}_{\alpha}+\mathrm{R}_{\beta}} .
$$

The directions $\alpha$ and $\beta$ are usually aligned with the main anisotropy axes of the sample surface plane. In our experiments, $\alpha$ and $\beta$ were chosen parallel to the [-110] and [110] directions on the (001) plane of the substrates (GaAs, InGaAs, see below for details), carefully aligned. RAS experiments were carried out on all the samples in the range $1.5 \div 4.5 \mathrm{eV}$ at near normal incidence using a home-made apparatus with two polarizers $[12,21]$. In all the reported spectra we have subtracted the structureless and nearly flat spectrum of an isotropic oxidised $\mathrm{Si}(001)$ sample measured in the same alignment condition, to eliminate the residual contributions coming from optics. For each spectrum we have then reported the corresponding "zero-line". At present no calculations exist about the anisotropic optical properties of $\mathrm{GaAsBi}$, helping to identify the different structures detected. Nevertheless, a careful phenomenological analysis of the whole series of spectra has allowed to reach stringent conclusions about the interpretation of our results.

GaAsBi samples have been grown by MBE using a solid-source MBE 32P Riber equipped with a conventional Knudsen effusion cell for $\mathrm{As}_{4}$, at the substrate temperature $T=325^{\circ} \mathrm{C}$, with a quasi3 
stoichiometric As-to-Ga flux ratio, and a growth rate of $0.5 \mathrm{ML} / \mathrm{s}$ for a thickness ranging from 60 to $170 \mathrm{~nm}$. To vary the Bi content four different Bi fluxes are used with a beam equivalent pressure in the range $1.3 \times 10^{-8}-1 \times 10^{-7} \mathrm{hPa}$. Semi-insulating GaAs(001) epiready substrates are used for all the samples. After standard cleaning and oxide removal procedures, an epitaxial GaAs buffer layer, $340 \mathrm{~nm}$ thick, is grown in As overflow at $590{ }^{\circ} \mathrm{C} . \mathrm{GaAs}_{1-\mathrm{x}} \mathrm{Bi}_{\mathrm{x}}$ films $(x=0.008-0.075)$ under compressive strain are successively grown on the GaAs buffer layer. $\mathrm{GaAs}_{1-x} \mathrm{Bi}_{\mathrm{x}}$ films $(x=0.007-0.072)$ under tensile strain are instead grown on top of a partially relaxed $\mathrm{In}_{y} \mathrm{Gaa}_{1-y} \mathrm{As} / \mathrm{In}_{z} \mathrm{Ga}_{1-z} \mathrm{As}(y=0.20-0.24, z=0.10-0.12)$ double layer (DL), with a thickness of $300 \mathrm{~nm}$ for each layer. The whole growth process is monitored in situ by RHEED. Further details of the samples growth and characterization are reported in ref.11.

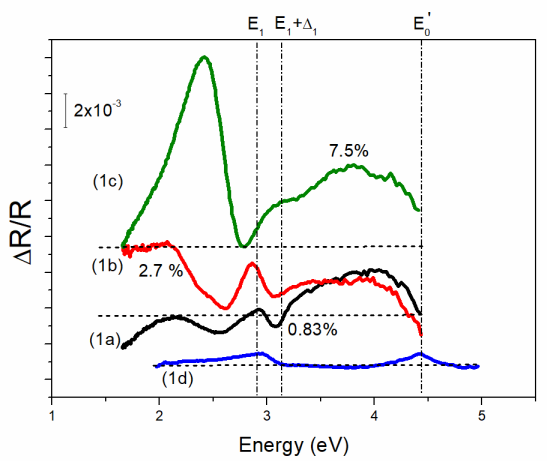

Fig.1 - RAS spectra of three GaAsBi samples grown by MBE under compressive strain, with increasing amount of Bi: 1a) $0.83 \%$ (black curve); 1b) $2.7 \%$ (red curve); 1c) $7.5 \%$ (olive curve). For the sign of RAS see text. Each curve has its own zeroline (curves $1 \mathrm{~b}$ and $1 \mathrm{c}$ refer to the same zeroline). The spectrum of a fully oxidized GaAs(001) surface is also reported (1d, blue curve). Photon energies corresponding to GaAs bulk critical points at room temperature have been highlighted with vertical dot-dashed lines.

In Fig. 1 and 2 we present and compare two groups of RAS spectra -corresponding to two different strain conditions-, measured on six (001) GaAsBi samples similarly oriented, but having different amounts of Bi concentration. All the spectra have been measured in air, on samples extracted from Ultra High Vacuum (UHV) after growth and then exposed to atmosphere for long time (several weeks). The three samples in Fig.1 (curves 1a,1b,1c) are nominally "compressive samples" (i.e, the substrate -onto which the GaAsBi layer has been grown- has a lattice parameter smaller than the growing layer) while the three 
samples in Fig.2 (curves 2a,2b,2c) are "tensile samples" (i.e, the substrate has a lattice parameter larger than the growing layer).

For comparison and reference in Fig. 1 we have also reported the RAS spectrum of an oxidised (001) GaAs sample, having the same orientation of the GaAsBi samples (1d, blue curve). A clean GaAs(001) surface was annealed in UHV to obtain an As-rich phase $2 \times 4$ reconstructed, then exposed to molecular oxygen to contaminate it purposely [18]. The increasing saturation of surface bonds by gas molecules progressively removes the surface-related anisotropies, until the final, stable curve is measured: it is quite flat (being reminiscent of the centrosymmetric and then isotropic bulk symmetry). Weak relic features appear at defined photon energies, that are the $E_{1}(2.91 \mathrm{eV}), \mathrm{E}_{1}+\Delta_{1}(3.13 \mathrm{eV})$ and $\mathrm{E}_{0}{ }^{\prime}(4.44 \mathrm{eV})$ bulk critical points energies. They are due to the modification of the bulk optical properties in consequence of the presence of an electric field perpendicular to the surface originating from the band-bending of bulk energy bands (Linear Electro Optic effect, LEO) [26].

We divide the spectral range into two regions:

i) below $3 \mathrm{eV}$ (low energy range);

ii) around and above $3 \mathrm{eV}$, and up to $4.5 \mathrm{eV}$ (high energy range).

The high energy range is characterized by anisotropies related to GaAsBi bulk, corresponding to $E_{1}, E_{1}+\Delta_{1}$ and $\mathrm{E}_{0}$ ' critical points (whose corresponding photon energies are highlighted in figures by vertical lines), due to LEO [26]. In our spectra $\mathrm{E}_{0}$ ' is slightly out of the measured range, but the slope of the spectral lineshape reaching its local extreme at $\mathrm{E}_{0}{ }^{\prime}$ is clearly visible. $\mathrm{E}_{1}$ and $\mathrm{E}_{1}+\Delta_{1}$ are instead well identified.

All these anisotropy features have a characteristic behaviour: according to the chosen sample orientation (the same in all experiments), in compressive (tensile) samples the signal has a local maximum (minimum) at $\mathrm{E}_{1}$, a local minimum (maximum) at $\mathrm{E}_{1}+\Delta_{1}$, and a downward (upward) slope at $\mathrm{E}_{0}$ '. The corresponding photon energies undergo a progressive red-shift with increasing Bi concentration, demonstrating the variation of the bulk critical points of GaAsBi with Bi insertion [27]. 


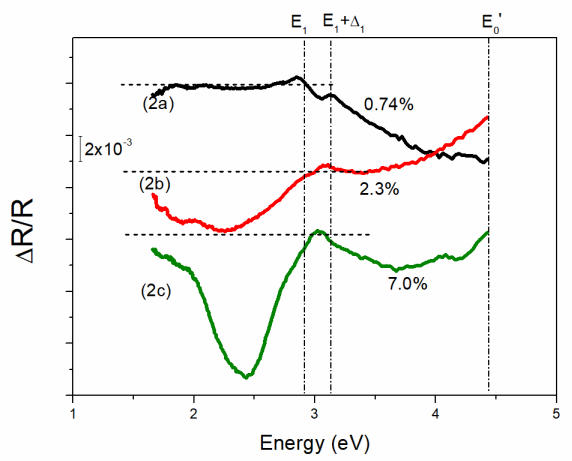

Fig. 2- RAS spectra of three GaAsBi samples grown by MBE under tensile strain, with increasing amount of Bi: 2a) $0.74 \%$ (black curve); 2b) $2.3 \%$ (red curve); 2 c) $7.0 \%$ (olive curve). For the sign of RAS see text.. Each curve has its own zeroline. Photon energies corresponding to GaAs bulk critical points have been highlighted with vertical dotdashed lines.

Below $3 \mathrm{eV}$ (a region where no critical points of the grown layer are present) the lineshape evolution with $\mathrm{Bi}$ concentration is dominated by the appearance of a structure (hereafter labeled as $\mathrm{A}$ ) centered in the energy range 2.2-2.5 eV. Its sign (according to the definition of RAS signal in our experiments) is negative (that is, polarized along the [110] direction on the surface), with one notable exception: in compressive samples, at the highest Bi concentration, it is positive. The amplitude of this structure has been evaluated for each spectrum with respect to its own zero-line, and the modulus values have been reported vs Bi concentration in fig. 3: the signal depends linearly upon Bi content, irrespective of the strain conditions (tensile or compressive).

What happens in region (ii) is well explained by the known progressive shift of bulk critical points energies towards lower photon energies with $B i$ concentration. The energies of $E_{1}$ and $E_{1}+\Delta_{1}$ transitions decrease monotonically, in qualitative agreement with published ellipsometry data [27]. On the contrary, the origin of the anisotropy reported in region (i) is not known, and how it is linked to Bi at the nanoscale. No theoretical calculations are available for the optical properties of these materials, but a conclusion is possible on the basis of the analysis of our data and phenomenological observations. 


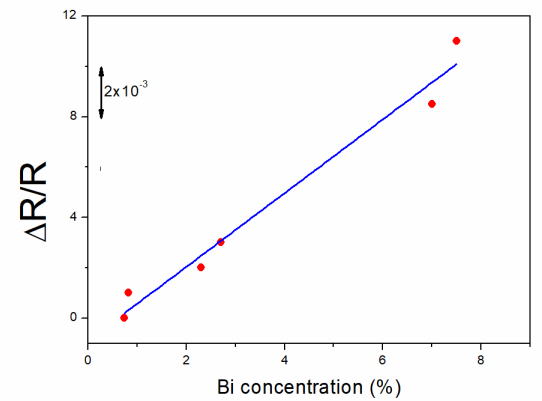

Fig. 3 - Modulus of the amplitude of the structure A (see text) visible in the low energy range of RAS spectra reported in Figs 1 and 2, versus Bi concentration. The values of the amplitude have been measured with respect to the zeroline of each RAS spectrum. A linear fit (blue line) is also shown. The vertical error bar $\left(2 \times 10^{-4}\right)$ is rendered by the size of the circle used for data points.

When GaAsBi is grown in the MBE chamber, by comparing its light absorption length [28] with the thickness of the layer (170 nm for all tensile samples; respectively $93 \mathrm{~nm}, 170 \mathrm{~nm}$ and $60 \mathrm{~nm}$ for the compressed series, from the lower to the higher $\mathrm{Bi}$ concentration) actually we conclude that at $3 \mathrm{eV}$ the RAS signal comes only from GaAsBi, as clearly demonstrated by the LEO structures, showing the red shift of the GaAsBi critical points with Bi concentration. At lower photon energies, on the contrary, more care is necessary, as in principle light could interrogate the substrate and the GaAsBi/substrate interface. However, also in this case we exclude contributions from the substrate, on the basis of the clear linear dependence of the structure A upon the concentration of Bi (an element present only in the GaAsBi layer) for the whole series of samples, irrespective of the lower thickness values. In addition, the possible contribution to RAS spectra from the GaAs substrate would be not significant: it is known that the (001)-terminated bulk is almost isotropic [12,13], apart from the region around $3 \mathrm{eV}$, where the absorption length value excludes any contribution.

The first issue to address is if this anisotropy is surface-related or bulk-related. As the spectra in figs 1 and 2 have been measured under atmospheric pressure condition, with samples extracted from UHV after growth and then stored in air for long time, a true surface origin - related to optical transitions between electronic states of the clean surface (as in GaAs(001) [18], InAs(001) [29, 30], C(111)2x1 [20], 
Si(111)2x1 [31]) must be ruled out. Further experiments conducted in UHV on ad-hoc grown samples will conclusively elucidate the origin of such anisotropy for $\mathrm{GaAsBi}$.

$\mathrm{GaAsBi}$ is anisotropic for the strain produced by large $\mathrm{Bi}$ atoms incorporated in the crystal structure, and related to well defined crystal directions, that are the bonding directions of $\mathrm{Bi}$ with neighboring atoms. It is known from literature that substitutional Bi atoms are not randomly distributed in the GaAs matrix, but correlate producing little clusters (dimers, triplets, tetramers) [6, 32-34]. However, when all the possible equivalent Bi-related structures coming from aggregated atoms (pairs, triplets and little clusters) are considered in the crystal, an overall total isotropy is expected on the average. On the contrary, during growth, structural and consequent electronic anisotropy are present in the surface layer, where directional bonds can form between adjacent Bi atoms, thus producing dimers [35]. When deposited on the surface, $\mathrm{Bi}$ atoms from MBE sources experience a well different situation with respect to the bulk: even the complete Bi coverage of a Ga-terminated surface layer (mimicking GaBi, a situation impossible in bulk) is obtained in case of the $2 \times 1$ reconstructed surface [35], with Bi dimers aligned along the [-110] direction and covering the whole layer. In (001) surfaces of III-V semiconductors, surface-related RAS structures have been measured and explained as connected to dimers [36].

We trace back the origin of the anisotropy signal A to the layers of the subsurface region (connected to the reconstruction of the surface layer and protected against contamination). In published papers buried Bi dimers [35] or even short chains of Bi atoms present below the surface [37] are reported. More generally we suppose the existence of directional bonds at the surface after deposition (dimers or chains of $\mathrm{Bi}$ atoms), then progressively and partly incorporated in the first layers below the surface when growth proceeds. In conclusion, RAS signal at about $2.4-2.5 \mathrm{eV}$ comes from the very last layers of the sample (not strictly from the surface), where a local anisotropic strain due to the atoms of Bi produces measurable effects in the optical spectrum.

The effect of strain in the optical anisotropy spectra has been carefully studied for III-V semiconductors, mainly focusing onto (001) surfaces. In III-Vs the otherwise isotropic bulk produces characteristic modifications of the spectral line at bulk critical points, depending upon applied stress, its direction, symmetry of the critical point, and doping (value, sign) of the sample. The evidence that in all (001) surfaces of these semiconductor anisotropy features are detected near or in coincidence with bulk 
critical points, plus the presence of dimers as building blocks of any proposed reconstruction model, suggests that directional surface bonds could cause local stress along the bonding direction (like "atomsized tweezers"), modifying the bulk optical properties in consequence of the existence and of the structure of the surface (bulk-modified-by-the-surface) [17,18]. This explanation cannot be extended "as it is" to our case, as there are no bulk critical points in the energy region we are considering. On the basis of our data, the RAS structure A implies that, although the microscopic mechanism is still unclear, electronic states localized below the surface are responsible for the measured anisotropy. They are related to structural and electronic modifications of the crystal matrix for the insertion of $\mathrm{Bi}$ atoms and the presence of directional bonds involving $\mathrm{Bi}$ atoms, thus producing the measured non-equivalence between [1-10] and [110] directions in the (001) surface plane.

A remarkable property of the Bi-related anisotropy is its change of sign at the $7 \%$ concentration, measured only for compressive samples. It is known that: i) RAS is sensitive to strain and in particular to the sign of strain; ii) compressive and tensile strain on a (001) surface result in axial strain with opposite sign $[38,39]$. On this ground, as the Bi-related structure sign is negative for low Bi quantities (without any difference between tensile and compressive samples), we conclude that the local strain experienced by GaAsBi in sample 1c (7\%, compressive) is different from all the other cases. In particular, at this concentration value for $\mathrm{Bi}$-that is the highest here reported-, the local situation is different when tensile and compressive samples are considered, as shown by the different sign of the A structure.

From this behavior of RAS sign, we propose that the final (and till now not yet determined) configuration of Bi atoms in high density samples (under tensile growth) is the same as in low density samples (in this case without distinguishing between tensile and compressive growth). We conclude that growth performed under tensile strain manages more efficiently a higher concentration of Bi atoms [11].

The same does not happen for compressive samples, where at high Bi concentration (at least equal to $7 \%$ ) the resulting strain produces a modification of the local structure necessary to relax the energy surplus coming from local deformation of the crystal. Information coming from other techniques (XRD, PL) confirm that sample 1c has a lower crystal quality (as labeled in terms of the optical behavior detected by PL), while at low Bi values compressive samples are of good quality [11]. The general trend suggests that - 
by the absolute sign of the structure at about $2.5 \mathrm{eV}$ - RAS monitors the "optical" fingerprint coming from the quality of the crystal, although at the moment the reason for that is unclear.

If comparing the signal amplitude of our spectra with amplitudes of experimental spectra reported in ref. 24 , almost equal values are obtained. This might mean correctly that the signal due to strain effects produced by $\mathrm{Bi}$ atoms dominate the spectrum measured during MOVPE growth. Further investigations and new experiments will be dedicated to study in UHV GaAsBi(001) surfaces.

In conclusion, we presented an experimental study by RAS of GaAsBi samples grown in MBE with increasing concentration of $\mathrm{Bi}$, up to the higher value of about $7 \%$, and different strain conditions (either compressive strain or tensile strain). A characteristic anisotropy signal below $2.5 \mathrm{eV}$ is connected to the presence of $\mathrm{Bi}$ and in particular to the local strain produced in the sub-surface region by the voluminous Bi atoms. Its amplitude directly relates to the Bi quantity, and its sign gives information about the local clustering/ordering of $\mathrm{Bi}$ atoms in the sub-surface region of the grown sample. This interpretation of RAS spectra offers the opportunity to utilize this technique to follow in real time the optical/electronic properties during the growth of GaAsBi in MBE and MOVPE.

\section{Acknowledgements.}

This study was partially supported by the projects "Consolidate the Foundation 2015 - BILLY" and "RSA2017 - Mission Sustainability" of the University of Rome Tor Vergata, the Bilateral Mobility Program between CNR (SAC.AD002.018.017) (Italy) and the Czech Academy of Sciences (CNR-16-02), and Sapienza University Ateneo funds 2020.

\section{REFERENCES}

[1] T. Tiedje, E. C. Young and A. Mascarenhas, Int. J. Nanotechnol. 5, 963 (2008).

[2] S. Tixier, M. Adamcyk and T. Tiedje, Appl. Phys. Lett. 822245 (2003).

[3] R. A. Simmons, S. R. Jin, S. J. Sweeney and S. K. Clowes, Appl. Phys. Lett. 107, 142401 (2015).

[4] H. Asahi, in: Kasap S., Capper P. (eds) Springer Handbook of Electronic and Photonic Materials Springer Handbooks. Springer, Cham., (2017)

10 
[5] C. Li, Z. Q. Zeng, D. S. Fan, Y. Hirono, J. Wu, T. A. Morgan, X. Hu, S. Q. Yu, Zh. M. Wang and G. J. Salamo, Appl. Phys. Lett. 99, 243113 (2011).

[6] H. Huang, J. Liu and W. Duan, Phys. Rev. B 90, 195105 (2014) .

[7] D. L. Sales, E. Guerrero, J. F. Rodrigo, P. L. Galindo, A. Yáñez, M. Shafi, A. Khatab, R. H. Mari, M. Henini, S. Novikov, M. F. Chisholm and S. I. Molina, Appl. Phys. Lett. 98, 101902 (2011).

[8] X. Lu, D. A. Beaton, R. B. Lewis, T. Tiedje and M. B. Whitwick, Appl. Phys. Lett. 92, 192110 (2008).

[9] K. Oe, J. Cryst. Gr. 237-239, 1481 (2002).

[10] R. B. Lewis, M. Masnadi-Shirazi and T. Tiedje, Appl. Phys. Lett. 101, 082112 (2012).

[11] E. Tisbi, E. Placidi, R. Magri, P. Prosposito, R. Francini, A. Zaganelli, S. Cecchi, E. Zallo, R. Calarco, E. Luna, J. Honolka, M. Vondráček, S. Colonna and F. Arciprete, Phys. Rev. Applied 14, 014028 (2020).

[12] P. Weightman, D.S. Martin, R.J. Cole and T. Farrell, Reports on Progress in Physics 68, 1251(2005).

[13] I. Kamiya, D. E. Aspnes, L. T. Florez and J. P. Harbison, Phys. Rev. B 46, 15894 (1992).

[14] H. Doscher, B. Kunert, A. Beyer, O. Supplie,K. Volz, W. Stolz and T. Hannappel, J.Vac. Sci. Tech. B 28, C5H1 (2010).

[15] J. Ortega-Gallegos, A. Lastras-Martínez, L. F. Lastras-Martínez and R. E. Balderas-Navarro, phys. stat. sol. (c) 5, 2573 (2008).

[16] C. Goletti, G. Bussetti, F. Arciprete, P. Chiaradia and G. Chiarotti, Phys. Rev. B 66, 153307 (2002).

[17] F. Arciprete, C. Goletti, E. Placidi, P. Chiaradia, M. Fanfoni, F. Patella, C. Hogan and A. Balzarotti, Phys. Rev. B 68, 125328 (2003).

[18] F. Arciprete, C Goletti, E Placidi, C Hogan, P Chiaradia, M Fanfoni, F Patella and A. Balzarotti, Phys. Rev. B 69, 081308 (2004).

[19] Ph. Hofmann, K. C. Rose, V. Fernandez, A. M. Bradshaw and W. Richter, Phys. Rev. Lett. 75, 2039 (1995).

[20] G. Bussetti, C Goletti, P Chiaradia and T Derry, EPL (Europhysics Letters) 79, 57002 (2007),

[21] C. Goletti, G. Bussetti, P. Chiaradia, A. Sassella and A. Borghesi, Appl. Phys. Lett. 83, 4146 (2003).

[22] A. Sassella, M. Campione, L. Raimondo, A. Borghesi, G. Bussetti, S. Cirilli, A. Violante, C. Goletti, and P. Chiaradia, Appl. Phys. Lett. 94, 073307 (2009).

11 
[23] B.G. Frederick, J.R. Power, R.J. Cole, C.C.Perry, Q.Chen, S.Haq, Th. Bertrams, N.Richardson and P.Weightman, Phys. Rev. Lett. 80, 4490 (1998).

[24] O. Maßmeyer, T. Hepp, R. Günkel, J. Glowatzki, W. Stolz and K. Volz, App. Surf. Sci., 533, 147401 (2020).

[25] A.Sassella, L.Raimondo, L.Fazi, S.Trabattoni, B.Bonanni, G.Bussetti and C.Goletti, Org. Ele. 62, 102 (2018).

[26] H. Tanaka, E. Colas, I. Kamiya, D. E. Aspnes and R. Bhat, Appl. Phys. Lett. 59, 3443 (1991).

[27] Z. L. Bushell, R. M. Joseph, L. Nattermann, P. Ludewig, K. Volz, J. L. Keddie and S. J. Sweeney, J. Appl. Phys. 123, 045701 (2018).

[28] M. Masnadi-Shirazi, R.B. Lewis, V. Bahrami-Yekta, T. Tiedje, M. Chicoine and P. Servati, J. Appl. Phys., 116, 223506 (2014).

[29] F Arciprete, C Goletti, S Almaviva and P Chiaradia, Surf. Sci. 515, 281 (2002).

[30] C Goletti, F Arciprete, S Almaviva, P Chiaradia, N Esser and W Richter, Phys. Rev. B 64, 193301 (2001).

[31] C Goletti, G. Bussetti, F Arciprete, P Chiaradia and G Chiarotti, Phys. Rev. B 66, 153307 (2002).

[32] G. Ciatto, E. C. Young, F. Glas, J. Chen, R. Alonso Mori and T. Tiedje, Phys. Rev. B 78, 035325 (2008).

[33] G. Ciatto, M. Thomasset, F. Glas, X. Lu and T. Tiedje, Phys. Rev. B 82, 201304 (2010).

[34] M. Wu, E. Luna, J. Puustinen, M. Guina and A.Trampert, Appl. Phys. Lett. 105, 041602 (2014).

[35] F.Bastiman, A.G.Cullis, J.P.R. David and S.J.Sweeney, J. Cryst. Growth 341, 19 (2012).

[36] A. I. Shkrebtii, N. Esser, W. Richter, W. G. Schmidt, F. Bechstedt, B. O. Fimland, A. Kley and R. Del Sole, Phys. Rev. Lett. 81, 721 (1998).

[37] A.Beyer, N. Knaub, P.Rosenow, K.Jandieri, P.Ludewig, L.Bannow, S.W.Koch, R.Tonner and K.Volz, Appl. Mat. Today 6, 22 (2017).

[38] L. F. Lastras-Martínez, M. Chavira-Rodríguez, R. E. Balderas-Navarro, J. M. Flores-Camacho and A. Lastras-Martínez, Phys. Rev. B 70, 035306 (2004).

[39] L. F. Lastras-Martinez, R. E. Balderas-Navarro, M. Chavira-Rodriguez, J. M. Flores-Camacho and A. Lastras-Martinez, phys. stat. sol. (b) 240, 500 (2003).

12 


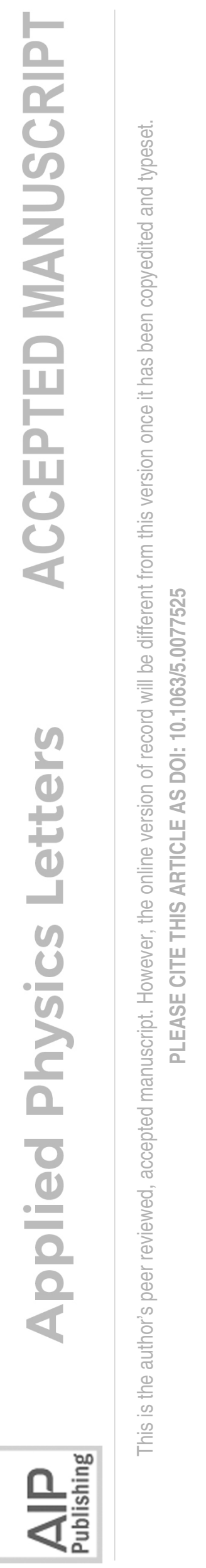




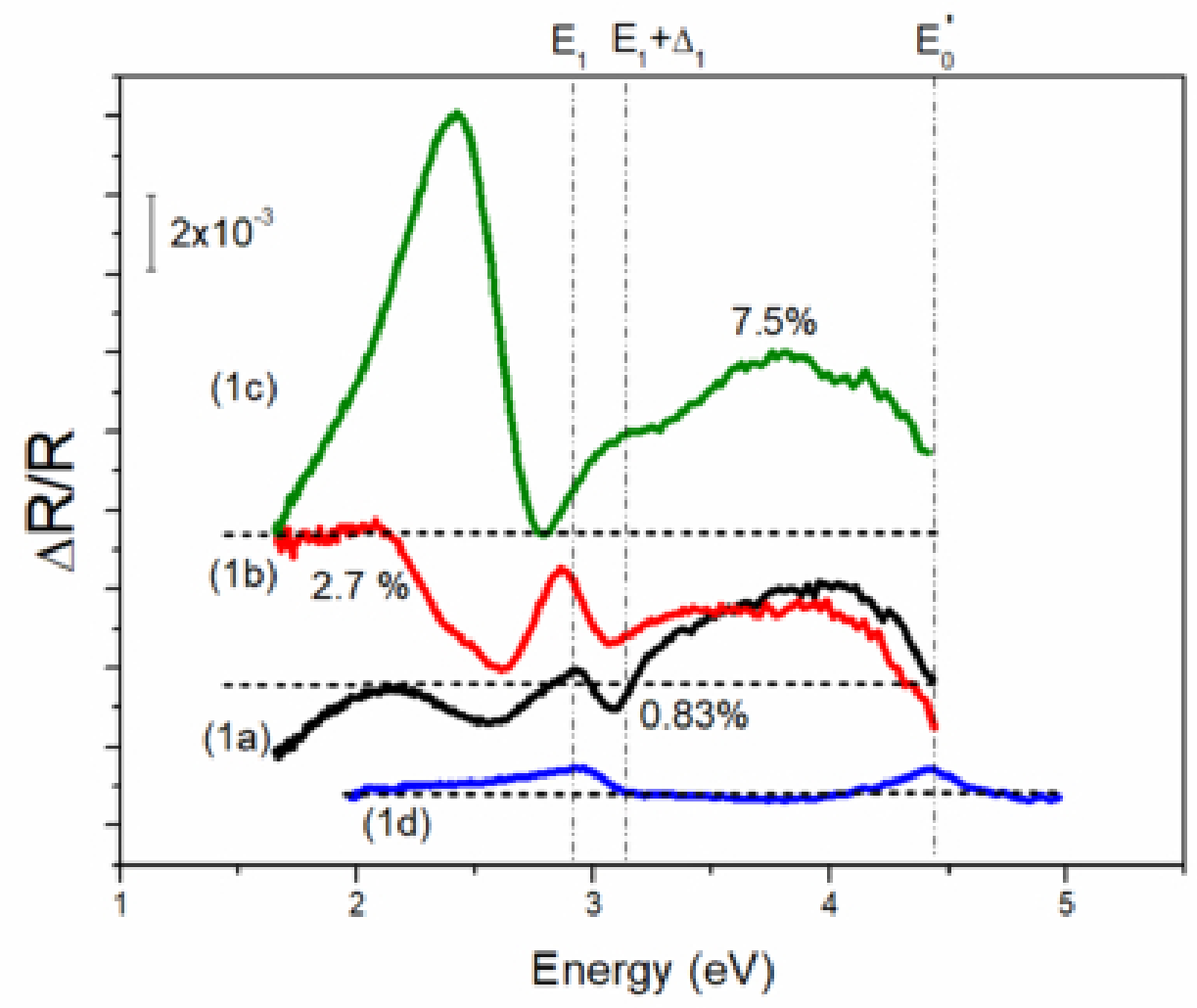

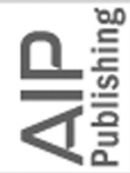




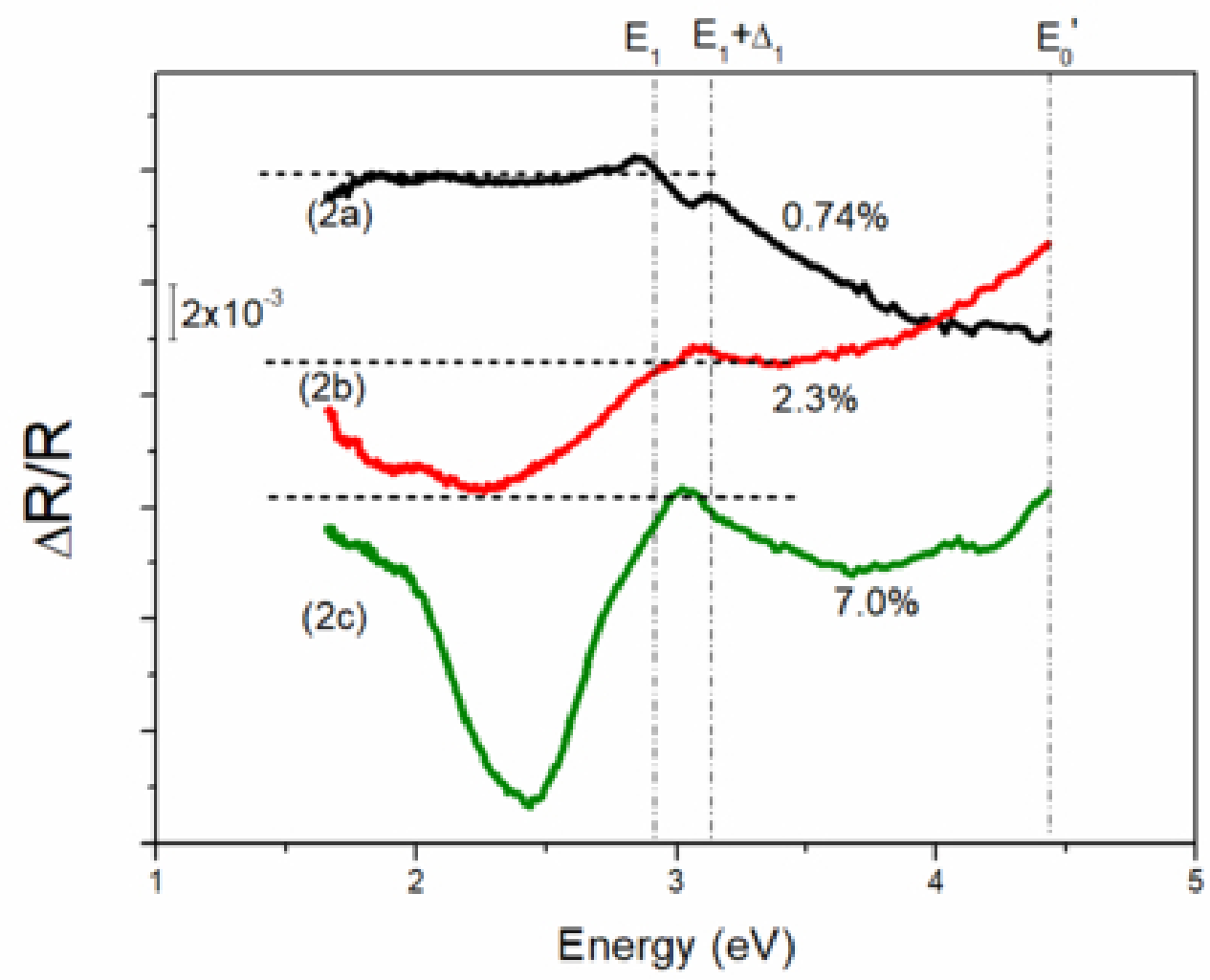

兄耪言 


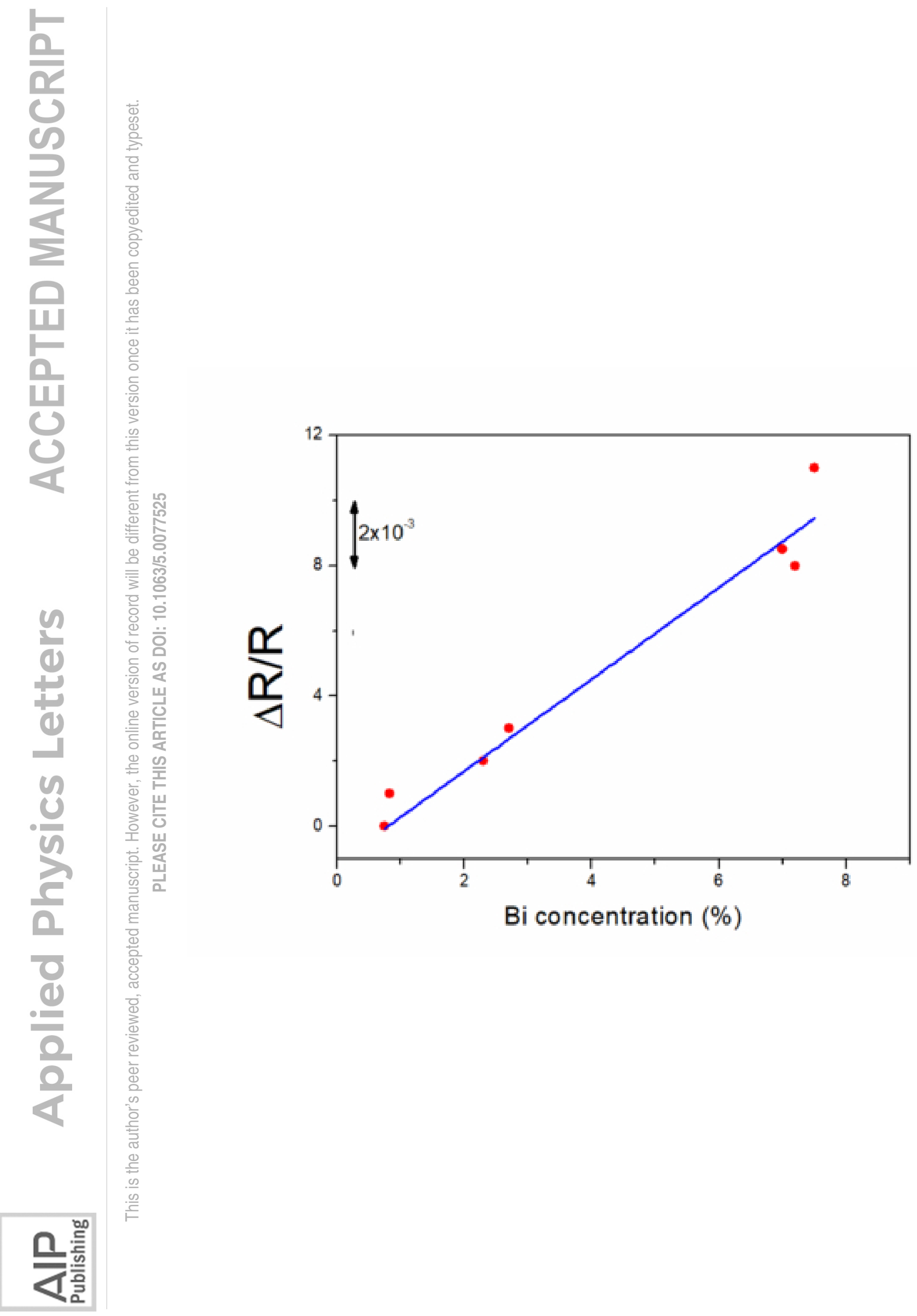

